\title{
High-flow therapy: physiological effects and clinical applications
}

Humidified high-flow therapy (HFT) is a noninvasive respiratory therapy, typically delivered through a nasal cannula interface, which delivers a stable fraction of inspired oxygen $\left(\mathrm{FlO}_{2}\right)$ at flow rates of up to $60 \mathrm{~L} \cdot \mathrm{min}^{-1}$. It is well-tolerated, simple to set up and ideally applied at $37^{\circ} \mathrm{C}$ to permit optimal humidification of inspired gas. Flow rate and $\mathrm{FIO}_{2}$ should be selected based on patients' inspiratory effort and severity of hypoxaemia. HFT yields beneficial physiological effects, including improved mucociliary clearance, enhanced dead space washout and optimisation of pulmonary mechanics. Robust evidence supports its application in the critical care setting (treatment of acute hypoxaemic respiratory failure and prevention of post-extubation respiratory failure) and emerging data supports HFT use during bronchoscopy, intubation and breaks from noninvasive ventilation or continuous positive airway pressure. There are limited data on HFT use in patients with hypercapnic respiratory failure, as an adjunct to pulmonary rehabilitation and in the palliative care setting, and further research is needed to validate the findings of small studies. The COVID-19 pandemic raises questions regarding HFT efficacy in COVID-19-related hypoxaemic respiratory failure and concerns regarding aerosolisation of respiratory droplets. Clinical trials are ongoing and healthcare professionals should implement strict precautions to mitigate the risk of nosocomial transmission.

\section{Educational aims}

- Provide a practical guide to HFT setup and delivery.

- Outline the physiological effects of HFT on the respiratory system.

- Describe clinical applications of HFT in adult respiratory and critical care medicine and evaluate the supporting evidence.

- Discuss application of HFT in COVID-19 and aerosolisation of respiratory droplets.
Cite as: D'Cruz RF, Hart N, Kaltsakas G. High-flow therapy: physiological effects and clinical applications. Breathe 2020; 16: 200224. 


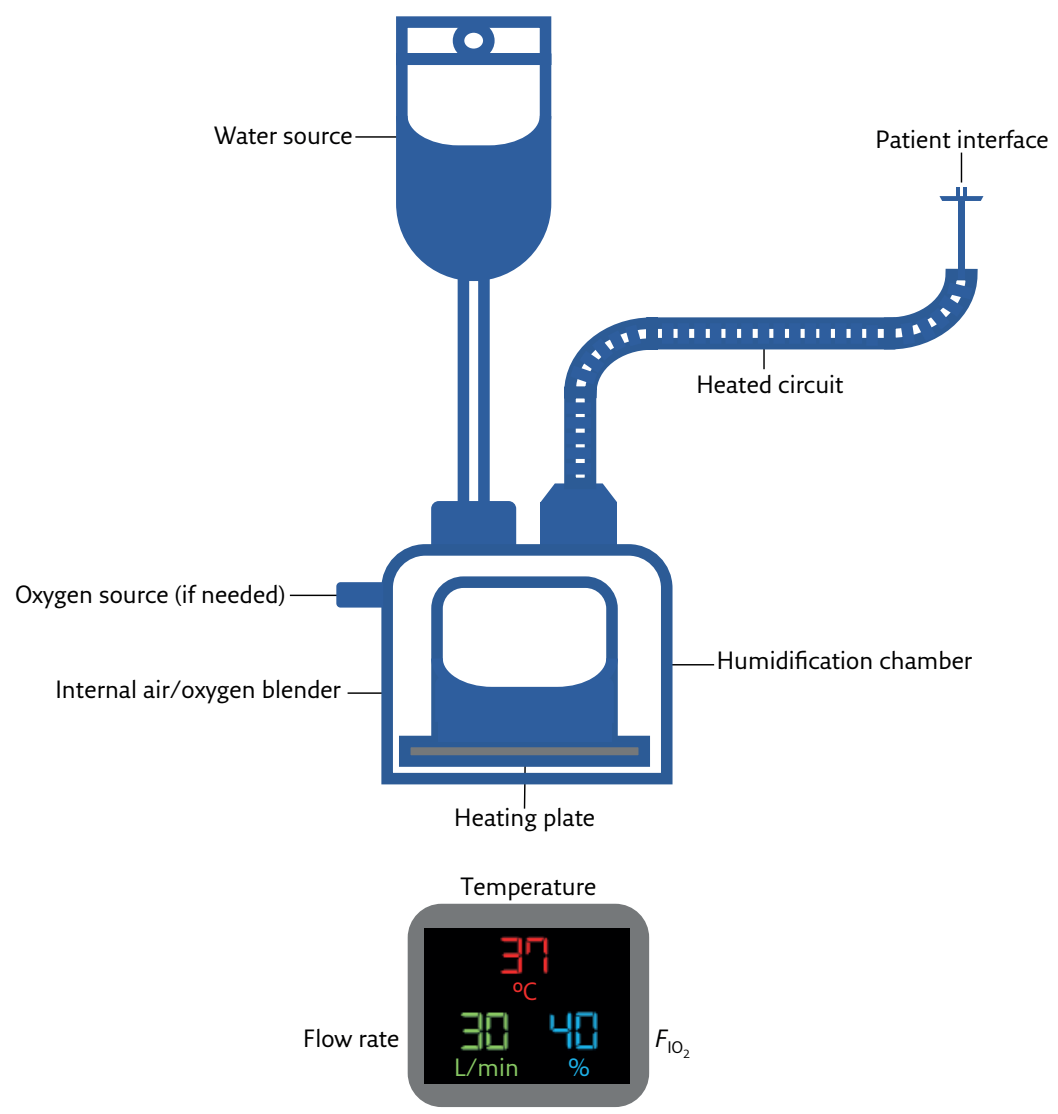

Figure 1 Schematic of HFT equipment and settings display: temperature (in ${ }^{\circ} \mathrm{C}$ ), flow rate (in $\mathrm{L} \cdot \mathrm{min}^{-1}$ ) and $\mathrm{FIO}_{2}$.

\section{A practical approach to setup and delivery}

HFT is most commonly delivered in the critical care setting; however, its ease of setup means that it may also be applied on high-dependency units and at home, following staff and patient training. The HFT system consists of an active heated humidifier, air/oxygen blender and heated single-limb circuit which can deliver gas at up to $60 \mathrm{~L} \cdot \mathrm{min}^{-1}$ (figure 1 ).

\section{Temperature}

Nasal passages are naturally conditioned to core body temperature, at which temperature inspired gas is humidified $100 \%$ relative to the atmosphere [2]. The ideal temperature setting for HFT is therefore $37^{\circ} \mathrm{C}$. Devices are typically able to set temperatures between $31^{\circ} \mathrm{C}$ and $39^{\circ} \mathrm{C}$ and temperatures below core body temperature may be used depending on patient preference.

\section{Flow}

HFT devices are capable of delivering maximum flow rates of $60 \mathrm{~L} \cdot \mathrm{min}^{-1}$. Careful observation is required to select a flow rate that matches the patient's maximal inspiratory flow. This avoids inhalation of excess ambient room air, thereby providing a stable and reliable fraction of inspired oxygen $\left(\mathrm{F}_{\mathrm{IO}}\right)$ to the alveoli [3]. Dilution of supplementary entrained oxygen may be unavoidable in patients in acute respiratory distress, who may breathe at flow rates exceeding $100 \mathrm{~L} \cdot \mathrm{min}^{-1}$ and in whom the maximal flow rate should be selected. masthod of warming and humidifying inspired gas which is delivered to patients under flow rates that exceed conventional oxygen delivery systems. This concept is referred to in many ways, including "humidified high-flow therapy (HHFT)", "nasal high-flow (NHF)", "high-flow nasal cannula (HFNC)" and "high-flow nasal oxygen (HFNO)", among others. Each term broadly refers to the same concept; however, since high-flow therapy does not necessarily require entrainment of oxygen, nor is it exclusively delivered through nasal cannula, we will adhere to the term "highflow therapy (HFT)".

HFT was first patented by Transpirator Technologies, Inc. in 1988 and was developed for use in both humans and racehorses to optimise their respiratory and cardiovascular function [1]. Commercial production soon followed, and in recent years, the range of clinical contexts in which HFT is applied continues to expand. In this review, we provide a practical guide to delivering HFT, describe its physiological effects on the respiratory system, summarise evidence-based clinical applications of HFT in adult respiratory and critical care medicine and discuss emerging HFT applications, where robust evidence is currently lacking.

\section{Oxygen}

Oxygen may be entrained through the HFT delivery system using the oxygen inlet port. In the hospital setting, piped oxygen should be used since oxygen cylinders are rapidly depleted when high flow rates are used. Following careful selection of flow rate to minimise entrainment of ambient air, the air/oxygen blender can reliably deliver an $\mathrm{FlO}_{2}$ of up to $100 \%$. HFT devices have built-in oxygen sensors which analyse inspired gas. This permits detection of $\mathrm{FlO}_{2}$ discrepancies between the device setting and what is delivered to the patient with an accuracy of $\pm 2-4 \%$.

\section{Interface}

HFT is most commonly delivered using a nasal cannula interface. The HFT nasal prongs are softer and more pliable than conventional nasal cannula and facemasks, making them more comfortable and better tolerated by patients [4]. Small, medium and large adult sizes are available. It is important to select a size that maximises the 
nasal prongs-to-nares ratio, as this impacts on the positive pressure delivered to the airways without detrimentally impacting on breathlessness or comfort [5]. HFT may also be delivered through a tracheostomy (provided the patient does not require mechanical ventilation), which may be achieved in hospital or in the domiciliary setting using commercially available adaptors.

\section{Consumables}

Humidification chambers, heated circuits, air inlet filters and patient interfaces are for single patient use and should be replaced regularly in accordance with manufacturers' guidance.

\section{Adverse events}

HFT is well-tolerated and stable patients report that it is more comfortable than noninvasive ventilation (NIV) and continuous positive airway pressure (CPAP) [6]. Patients in acute hypoxaemic respiratory failure report that HFT is additionally associated with less mouth dryness and improved breathlessness than facemask oxygen [4]. No serious adverse events have been reported in the literature as a consequence of HFT [7]. Prolonged use may cause localised skin damage (erythema and skin breakdown) in the nasal philtrum and beneath the straps, however this risk is substantially lower than with NIV or CPAP $[8,9]$. Patients and clinicians should regularly monitor for skin changes and adjust the interface size, strap tightness and/or HFT settings accordingly. When initiating HFT, patients may initially find the flow or temperature uncomfortable, however this sensation is typically limited to the first few minutes of setup before resolving entirely [4]. A period of acclimatisation, where the patient is commenced on a sub-therapeutic flow rate that is slowly titrated up to the desired level during close bedside observation, enables familiarisation with the HFT sensation and identification of appropriate flow and temperature settings.

The clinical indication for HFT delivered in the acute setting should be documented clearly and patients must be monitored for signs of treatment failure, an approach which also applies to the delivery of NIV and CPAP. Such signs may include deteriorating oxygen saturation, and increased respiratory rate and work of breathing. Continuous physiological monitoring and regular clinical review reduces the risk of delaying invasive mechanical ventilation, which is associated with a poor prognosis.

\section{Physiological effects on the respiratory system}

HFT produces a number of physiological changes in the respiratory system when delivered through a nasal interface, which are summarised in table 1.

\section{Mucociliary clearance}

The mucociliary transport system extends from the bronchioles to the nasopharynx and acts as a mechanical defence by which contaminants are trapped and transported out of the airways. The respiratory epithelium functions most effectively at core body temperature and $100 \%$ relative humidity, and is highly sensitive to changes in airway temperature and pressure. Extremes of humidity or a fall in airway temperature below core body temperature alters the viscosity of respiratory secretions, precipitates bronchoconstriction, reduces cilia beating frequency and reduces mucociliary clearance velocity [2]. Such changes occur during conventional oxygen delivery, where the cool, dry gas alters the physical and biochemical properties of respiratory mucus and may cause bronchoconstriction, particularly in patients with sensitive airways, such as those with asthma and COPD [10, 11]. Impaired mucociliary clearance reduces lung compliance, increases airway resistance and increases work of breathing, and is associated with increased risk of mucus plugging, alveolar derecruitment and infection. Effective secretion clearance is of particular importance for patients with COPD or bronchiectasis, where chronic mucus hypersecretion predisposes to recurrent respiratory infection and chronic bacterial colonisation, is associated with lung function decline and increases risk of hospitalisation [12, 13]. Sputum clearance is notoriously challenging to quantify directly and techniques employed in clinical practice have marked limitations, including inaccuracy of expectorated sputum measurement and subjectivity of patient-reported descriptors. Surrogate end-points may be employed and radioaerosol lung deposition and clearance studies reveal that tracheobronchial secretion clearance significantly increases following inhalation of air that is fully saturated and conditioned to $37^{\circ} \mathrm{C}$ in patients with obstructive airways disease [14].

\section{Dead space washout}

Experiments on lung models demonstrate that delivery of gas under high flow rates through a nasal

Table 1 Physiological effects of humidified high-flow therapy

\begin{tabular}{ll}
\hline Physiological effect & Impact \\
\hline Mucociliary clearance & $\begin{array}{l}\text { Optimised respiratory epithelial function } \\
\text { Improved mucociliary transport in bench studies }\end{array}$ \\
Dead space washout & $\begin{array}{l}\text { Reduced anatomical dead space } \\
\text { Carbon dioxide washout and oxygen reservoir }\end{array}$ \\
Improved pulmonary & Reduced work of breathing \\
mechanics & Increased lung compliance \\
& Improved gas exchange \\
& Improved ventilation homogeneity
\end{tabular}


cannula enables continuous flushing of exhaled gases from the upper airways and creates an oxygen reservoir. This wash out of anatomical dead space increases in proportion to increasing flow rate and reduces rebreathing of exhaled carbon dioxide [15]. In COPD, dead space contributes to exercise intolerance and impaired ventilation, and delivery of high flow gas has been shown to increase endurance and oxygen saturation during exercise [16]. The high flow rates that can be achieved with HFT also facilitates the delivery of a stable $\mathrm{FlO}_{2}$ that closely matches the prescribed $\mathrm{FIO}_{2}$, reliably increases with increasing flow rate and is further increased by mouth breathing, probably due to the reservoir effect [17].

\section{Pulmonary mechanics}

HFT is an open system meaning that, unlike with NIV, CPAP and invasive mechanical ventilation, there is not a closed circuit between the device and the patient, and gas leak from the mouth is expected. The positive airway pressure delivered by $\mathrm{HFT}$ is therefore low, typically under $3 \mathrm{cmH}_{2} \mathrm{O}$ with the mouth closed and $1 \mathrm{cmH}_{2} \mathrm{O}$ with the mouth open when delivered at $35 \mathrm{~L} \cdot \mathrm{min}^{-1}$, and increases by $0.7 \mathrm{~cm} \mathrm{H}_{2} \mathrm{O}$ for every $10 \mathrm{~L} \cdot \mathrm{min}^{-1}$ increase in flow rate [18]. The increase in mean positive airway pressure, in combination with mucociliary clearance, dead space washout and stable $\mathrm{FIO}_{2}$ probably explain the following changes in pulmonary mechanics that are observed during HFT delivery.

\section{1) Reduced work of breathing}

Inspiratory effort and metabolic work of breathing may be quantified using oesophageal balloon

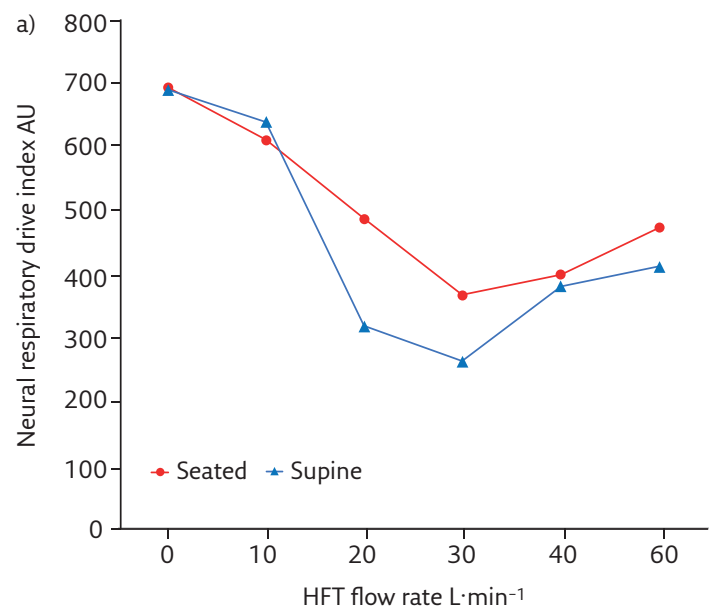

catheters to measure transdiaphragmatic pressure $\left(P_{\mathrm{di}}\right)$ and the diaphragm pressure-time product $\left(\mathrm{PTP}_{\mathrm{di}}\right)$. In acute hypoxaemic respiratory failure, compared with facemask oxygen, HFT has been shown to improve lung compliance and reduce inspiratory effort and work of breathing [19]. The positive pressure delivered by HFT also appears sufficient to counterbalance the threshold load imposed by hyperinflation in COPD, as demonstrated by reduced intrinsic positive endexpiratory pressure $\left(P E E P_{i}\right)$, inspiratory effort and work of breathing compared with baseline [20]. Parasternal electromyography $\left(\mathrm{EMG}_{\mathrm{para}}\right)$ has also been used to demonstrate changes in inspiratory muscle activity in stable COPD when HFT is delivered at different flow rates (figure 2).

\section{2) Improved lung volumes and ventilation}

In healthy volunteers, stable COPD, pulmonary fibrosis and in critical care, HFT is associated with a reduced respiratory rate, stable or increased tidal volumes and improved breathlessness compared with conventional low-flow oxygen and facemask oxygen [21]. HFT also improves ventilation homogeneity (measured using electrical impedance tomography) and oxygenation [19]. In hypercapnic COPD patients, home NIV remains the standard of care both in stable patients and those with persistent hypercapnia following a severe exacerbation requiring acute NIV [22, 23]. Whilst small physiological studies demonstrate that HFT delivered over 20-30 min to hypercapnic COPD patients on home oxygen can reduce arterial and transcutaneous carbon dioxide, the clinical

b)

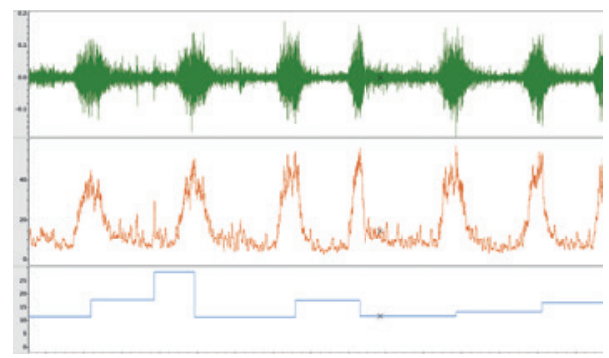

c)

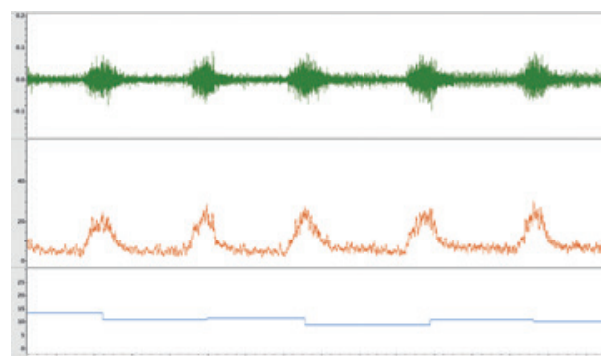

Figure 2 Changes in neural respiratory drive (quantified using EMG ${ }_{\text {para }}$ ) in a patient with stable COPD. a) Neural respiratory drive quantified with $E M G_{\text {para }}$ in a patient with very severe COPD (forced expiratory volume in $1 \mathrm{~s}<30 \%$ predicted). Neural respiratory drive index (the product of normalised $E M G_{\text {para }}$ and respiratory rate, $A U=$ arbitrary units) was measured at baseline and with HFT delivered at 10, 20, 30, 40 and $60 \mathrm{~L} \cdot \mathrm{min}^{-1}$ at $37^{\circ} \mathrm{C}, \mathrm{F}_{\mathrm{OO}_{2}} 0.21$. Panels b) and c) illustrate raw $E M G_{\text {para }}\left(m V\right.$, green), root mean square $E M G_{\text {para }}\left(\mu \mathrm{V}\right.$, orange) and respiratory rate (breaths $\cdot \mathrm{min}^{-1}$, blue). b) High neural respiratory drive observed at baseline. c) Optimal offloading of the respiratory muscle pump observed at $30 \mathrm{~L} \cdot \mathrm{min}^{-1}$, with a significant reduction in neural respiratory drive. 
implications of these data are not established and NIV remains the gold standard in managing hypercapnic respiratory failure in this cohort $[20,21]$.

Interestingly, when HFT is applied through tracheostomies, during weaning from invasive mechanical ventilation, there are no differences in respiratory rate, gas exchange, work of breathing or neural respiratory drive compared with conventional oxygen, suggesting that the beneficial physiological effects of HFT are mediated principally through changes to the upper airway [24].

\section{Evidence-based clinical applications}

The strongest evidence base for HFT is in the management of acute hypoxaemic respiratory failure. Other indications where there are robust supportive data include prevention of postextubation respiratory failure, breaks from positive airways pressure therapy and pre-procedure preoxygenation (table 2).

\section{Hypoxaemic respiratory failure}

The five principal causes of hypoxaemia are ventilation/perfusion mismatch, hypoventilation, diffusion limitation, right-to-left shunt and reduced inspired oxygen tension. Oxygen is the mainstay of therapy and high flow rates may be required during acute illness. Of note, in hypoxaemia caused by hypoventilation, ventilation must be administered (with or without oxygen, as appropriate). Conventional oxygen delivery methods are limited in the flow rate that can be delivered: nasal cannula may deliver up to $6 \mathrm{~L} \cdot \mathrm{min}^{-1}$, simple facemasks up to $10 \mathrm{~L} \cdot \mathrm{min}^{-1}$, and Venturi masks and nonrebreather masks up to $15-30 \mathrm{~L} \cdot \mathrm{min}^{-1}$ of oxygen.
Patients in acute hypoxaemic respiratory failure typically breathe at flow rates of $60-100 \mathrm{~L} \cdot \mathrm{min}^{-1}$, which leads to ambient air inhalation and dilution of the $\mathrm{FlO}_{2}$ delivered to the alveoli. Additionally, conventional oxygen delivery systems may not be heated or humidified which can lead to drying of the mouth, upper airway and respiratory secretions, mucosal ulceration and epistaxis [4]. As previously described, HFT permits delivery of humidified gas at flow rates closer to patients' inspiratory effort, thereby delivering a more reliable $\mathrm{FIO}_{2}$.

Several randomised studies have demonstrated that oxygen delivered through HFT is safe and effective in the management of acute hypoxaemic respiratory failure. The largest of these was conducted by FRAT et al. [25] who randomised patients with a $\mathrm{PaO}_{2}: \mathrm{FIO}_{2} \leq 300$ to receive $\mathrm{HFT}$, facemask oxygen or NIV. This study identified that the difference in intubation rates observed between the three interventions (38\%, 47\% and 50\%, respectively) did not reach statistical significance. However, in patients with moderate or severe hypoxaemia $\left(\mathrm{PaO}_{2}: \mathrm{FIO}_{2} \leq 200\right)$, facemask oxygen and NIV were associated with increased risk of intubation compared with HFT, and in-ICU and 90-day mortality were lower in the HFT group. Additionally, HFT was associated with a greater improvement in breathlessness within the first hour of treatment.

The clinical benefits of HFT in acute hypoxaemic respiratory failure do not appear to be conferred to immunocompromised patients, including those with iatrogenic immunosuppression (e.g. steroids, chemotherapy, transplant anti-rejection drugs), haematological malignancy or primary immunodeficiency. A randomised trial performed by Azoulay et al. [30] demonstrated no differences in 28- and 90-day mortality, intubation rate, ICU or hospital length of stay, comfort or breathlessness between immunocompromised patients who received HFT or facemask oxygen, despite HFT being associated with reduced respiratory rate

Table 2 Evidence-based indications for humidified high-flow therapy

\begin{tabular}{|c|c|}
\hline Clinical indication & Effects \\
\hline \multirow{4}{*}{$\begin{array}{l}\text { Acute hypoxaemic respiratory } \\
\text { failure }[8,25]\end{array}$} & Similar rates of intubation compared with NIV and facemask oxygen \\
\hline & Reduced risk of intubation in patients with moderate or severe hypoxaemia $\left(\mathrm{PaO}_{2}: \mathrm{FIO}_{2} \leq 200\right)$ \\
\hline & Reduced ICU and 90-day mortality \\
\hline & Improved breathlessness following treatment initiation \\
\hline \multirow{2}{*}{$\begin{array}{l}\text { Prevention of post-extubation } \\
\text { respiratory failure }[9,26]\end{array}$} & Low-risk patients: HFT superior to conventional oxygen \\
\hline & High-risk patients: HFT noninferior to NIV \\
\hline \multirow{2}{*}{$\begin{array}{l}\text { Breaks from positive airway } \\
\text { pressure [27] }\end{array}$} & Permits oral intake (medication, nutrition) and communication \\
\hline & More comfortable than conventional nasal cannula and NIV \\
\hline \multirow{2}{*}{$\begin{array}{l}\text { Oxygenation during airway } \\
\text { procedures }[28,29]\end{array}$} & Pre-oxygenation prior to and during endotracheal intubation \\
\hline & Improves oxygenation during bronchoscopy compared with conventional nasal cannula \\
\hline
\end{tabular}

$\mathrm{PaO}_{2}$ : arterial oxygen tension; ICU: intensive care unit. 
and a sustained improvement in oxygenation. Reasons for these findings remain speculative, and may relate to the overall lower survival in this population compared with immunocompetent patients with acute hypoxaemic respiratory failure [31]. A holistic approach should therefore be adopted when managing immunocompromised patients, involving treatment of the underlying cause of respiratory failure, prompt identification of multisystem complications and management of preexisting comorbidities, in addition to correction of hypoxaemia.

\section{Prevention of post-extubation respiratory failure}

Prior to planned extubation, patients are categorised as high- or low-risk of reintubation based on duration of mechanical ventilation, severity of critical illness, secretion burden and cough strength, upper airway patency concerns, comorbidities and age [32]. Two randomised trials led by HERNANDEZ et al. [9, 26] demonstrated that HFT was superior to conventional oxygen (nasal cannula or facemask) in low-risk patients and noninferior to NIV in high-risk patients in preventing post-extubation respiratory failure in a general ICU cohort, and ICU length of stay was shorter in those receiving HFT compared with those receiving NIV post-extubation. Similarly, HFT has been identified as noninferior to NIV in preventing post-extubation respiratory deterioration in post-operative cardiothoracic surgical patients, who are at high risk of postoperative respiratory failure [8]. In-ICU mortality, breathlessness and comfort were also similar between the two groups in this study.

\section{Breaks from positive airway pressure therapy}

NIV and CPAP are first-line treatments in acute hypercapnic respiratory failure and acute cardiogenic pulmonary oedema, respectively, and are associated with reduced intubation rate, mortality and hospital length of stay [33, 34]. However, these techniques are widely regarded by patients as uncomfortable and claustrophobic and pressure-related skin ulceration from the mask interface is common [9]. Following initiation of acute NIV or CPAP and attainment of relative physiological stability (improved work of breathing, resolution of acidosis, arterial partial pressure of carbon dioxide and oxygen $\left(\mathrm{PaCO}_{2}\right.$ and $\left.\mathrm{PaO}_{2}\right)$ within acceptable parameters), it is reasonable to permit NIV/CPAP breaks for intake of oral nutrition and medication and to assist verbal communication. During these breaks, oxygen may be administered using HFT, since this is better tolerated than conventional nasal cannula, although there is no evidence that this approach reduces total duration of positive airway pressure therapy [27].

\section{Oxygenation during airway procedures}

Prior to elective and emergency endotracheal intubation, pre-oxygenation is conventionally performed with a bag-valve-mask and reservoir bag which is removed immediately prior to an intubation attempt, during which time derecruitment and desaturation can occur. Nasal HFT enables oxygen delivery throughout the intubation process and improves oxygenation before, during and after the procedure [28].

Patients undergoing elective or emergency bronchoscopy are at risk of desaturation, particularly in the presence of chronic respiratory disease and pharmacological sedation administered for procedure tolerance. In these patients, oxygen may be delivered throughout and HFT has been shown to improve pre- and peri-procedure oxygenation compared with conventional nasal cannula [29].

\section{Speculation on emerging applications}

HFT application in both the acute and home settings has increased in recent years, sometimes without clear evidence to support the clinical indication. In this section, we review settings where the evidence supporting HFT is more limited and discuss potential risks that may arise.

\section{Hypercapnic respiratory failure}

Hypercapnic respiratory failure arises as a consequence of imbalance in the loads, capacity and drive of the respiratory muscle pump. There is substantial, robust evidence demonstrating that NIV improves a range of clinical and physiological outcomes in acute and chronic hypercapnic respiratory failure in obstructive lung disease, neuromuscular disease and obesity hypoventilation syndrome [35]. HFT is not a method of delivering ventilation and should not be applied to patients who require positive pressure ventilation. Physiological studies indicate that HFT can reduce $\mathrm{PaCO}_{2}$ in stable COPD and pulmonary fibrosis patients when delivered over short periods [20, 36], and a clinical trial comparing HFT to NIV in stable hypercapnic COPD demonstrated noninferiority of HFT in reducing $\mathrm{PaCO}_{2}$ after 6 weeks of treatment and higher adherence in the HFT group [37]. However, medium- and long-term clinical effects (exacerbation frequency, hospitalisation, mortality) of HFT used in this context remain unknown. While studies evaluating HFT in acute 
and acute-on-chronic hypercapnic respiratory failure in COPD are currently underway [38, 39], clinicians must continue to advocate NIV as firstline therapy in this cohort.

\section{Domiciliary use}

Domiciliary HFT may be considered for patients established on home oxygen for chronic hypoxaemic respiratory failure. There is evidence that regular nightly and/or daily use is associated with reduced exacerbation frequency and hospitalisation rate and improves breathlessness, exercise tolerance and quality of life compared with conventional home oxygen in COPD patients [40].

\section{Exercise adjuvant}

When delivered to stable COPD patients undergoing exercise testing on a cycle ergometer, HFT may enable patients to exercise for longer, with less breathlessness and improved oxygenation compared with conventional low-flow oxygen [16], benefits which are not apparent in patients recovering from an exacerbation [41]. HFT may be beneficial when delivered throughout a pulmonary rehabilitation programme, and has been shown to improve exercise tolerance, hyperinflation (measured using inspiratory capacity), breathlessness and quality of life in patients recovering from a COPD exacerbation compared with patients completing the programme without HFT, with no differences in exacerbation frequency or number of hospitalisations observed at 1 year [42].

\section{Palliative care}

Robust data guiding the use of HFT in patients with established ceilings of care and those with terminal breathlessness are lacking [43]. HFT may be considered to provide palliative support to patients with acute respiratory failure where mechanical ventilation has failed, is withdrawn or considered clinically inappropriate [44]. Multidisciplinary team collaboration with the patient should be used to guide decision-making, balancing potential benefits of symptom relief against risks of discomfort from the device and prolonging suffering.

\section{High-flow therapy during the COVID-19 pandemic}

Since the categorisation of novel severe acute respiratory syndrome coronavirus 2 (SARSCoV-2/COVID-19) as a pandemic on 11 March 2020, confirmed cases have continued to rise rapidly worldwide. Patients typically present with fever, breathlessness and cough and may develop profound hypoxaemic respiratory failure. Preliminary observations indicate that HFT may be an effective first-line therapy in patients

\section{Self-evaluation questions}

1. HFT has the following physiological effects:
a) Reduced work of breathing
b) Dead space washout
c) Improved mucociliary clearance
d) All of the above

2. Commonly reported side-effects of HFT include:
a) Nasal bridge ulceration
b) Pneumothorax
c) Temperature-related discomfort
d) Epistaxis

3. For patients in acute hypoxaemic respiratory failure treated with HFT, the flow rate should be set:
a) at $20 \mathrm{~L} \cdot \mathrm{min}^{-1}$
b) at $30 \mathrm{~L} \cdot \mathrm{min}^{-1}$
c) at $60 \mathrm{~L} \cdot \mathrm{min}^{-1}$
d) based on patients' inspiratory flow

4. Which of the following is not an evidence-based clinical application of HFT?
a) Acute hypoxaemic respiratory failure
b) Acute hypercapnic respiratory failure
c) Prevention of post-extubation respiratory failure
d) Pre-oxygenation prior to intubation

with mild-to-moderate hypoxaemic respiratory failure $\left(\mathrm{PaO}_{2}: \mathrm{FlO}_{2}>100 \mathrm{mmHg}\right)$ and the results of multicentre clinical trials evaluating oxygen delivery methods (HFT, CPAP and low-flow oxygen), such as RECOVERY-RS (ISRCTN16912075; https:// doi.org/10.1186/ISRCTN16912075) are eagerly awaited [45]. Treatment of severe COVID-19 pneumonitis likely requires a high $\mathrm{FIO}_{2}$. When oxygen is delivered at high flow rates using HFT, local oxygen supplies may be rapidly depleted, and this must be considered when preparing and allocating resources to manage large numbers of patients during a pandemic.

Concerns have been raised regarding aerosolisation of respiratory droplets and risks of nosocomial transmission of COVID-19 to healthcare workers. Lung model studies indicate that air dispersion distances with HFT are reasonably small, increasing with increasing flow rate and decreasing with worsening lung injury (between $3 \mathrm{~cm}$ delivered at $10 \mathrm{~L} \cdot \mathrm{min}^{-1}$ in severe lung injury and $17 \mathrm{~cm}$ delivered at $60 \mathrm{~L} \cdot \mathrm{min}^{-1}$ to a normal lung) [46]. However, this distance is likely to increase during coughing, with a maximum cough distance of $4.5 \mathrm{~m}$ reported in healthy volunteers receiving HFT [47]. The risk of COVID-19 transmission to healthcare workers appears to be low [45]; however, the proportions of healthcare workers infected with SARS in 2003 who were exposed to patients receiving HFT, NIV and invasive mechanical ventilation were reported to be $8 \%, 38 \%$ and $35 \%$, respectively, in a retrospective observational study [48]. It is therefore sensible to implement 
precautions to mitigate risks to healthcare professionals during HFT delivery, including strict use of personal protective equipment, regular handwashing, prompt disposal of condensate, use of negative pressure rooms where practical and covering the patient's nose and mouth with a mask.

\section{Conclusion}

Humidified HFT is a simple and well-tolerated method of delivering a stable $\mathrm{FlO}_{2}$ at flow rates of up to $60 \mathrm{~L} \cdot \mathrm{min}^{-1}$. HFT yields beneficial physiological effects, including improved mucociliary clearance, dead space washout and improved pulmonary mechanics. There is a strong evidence base for its use in the critical care setting and evidence-based applications include during NIV or CPAP breaks and during intubation or bronchoscopy. There is a theoretical risk of aerosolisation of respiratory droplets during HFT delivery and, during the COVID-19 pandemic, healthcare professionals must implement strict precautions to mitigate the risk of nosocomial transmission.

\section{Key points}

- HFT involves active heating and humidification of inspired gas from an air/ oxygen blender and its delivery to the patient through a heated circuit at a flow rate up to $60 \mathrm{~L} \cdot \mathrm{min}^{-1}$.

- Physiological effects of HFT include improved mucociliary clearance, dead space washout and reduced work of breathing.

- HFT is associated with demonstrable clinical and physiological benefits in acute hypoxaemic respiratory failure, prevention of post-extubation respiratory failure, oxygenation during airway procedures (intubation, bronchoscopy) and during breaks from positive airway pressure therapy.

- There are limited data on HFT in acute hypercapnic respiratory failure, in the home, as an adjuvant to pulmonary rehabilitation and in the palliative care setting, and prospective clinical trials to guide clinical practice are warranted.

- Results of clinical trials evaluating the effects of HFT in COVID-19 are awaited. Respiratory droplets may be aerosolised during HFT delivery and healthcare professionals must take appropriate precautions to mitigate risk of nosocomial transmission.

\section{Affiliations}

\section{Rebecca F. D'Cruz ${ }^{1,2}$, Nicholas Hart ${ }^{1,2}$, Georgios Kaltsakas ${ }^{1,2}$}

${ }^{1}$ Lane Fox Clinical Respiratory Physiology Research Centre, Guy's and St Thomas' NHS Foundation Trust, London, UK. ${ }^{2}$ Centre for Human \& Applied Physiological Sciences, King's College London, London, UK.

\section{Conflict of interest}

R.F. D'Cruz has nothing to disclose. N. Hart reports grants from Philips-Respironics (OPIP Trial, unrestricted research grant), RESMED (HoT-HMV Trial, unrestricted research grant) and Philips-Respironics (HoT-HMV Trial, unrestricted research grant), non-financial support from Philips-Respironics RT Meeting (development of MYOTRACE technology), outside the submitted work. In addition, Prof. Hart has a European patent for MYOTRACE issued, and a US patent for MYOTRACE pending. Prof. Hart's research group has received unrestricted grants (managed by Guy's \& St Thomas' Foundation Trust) from Philips and Resmed. Philips are contributing to the development of the MYOTRACE technology. G. Kaltsakas has nothing to disclose. 


\section{References}

1. Transpirator Technologies, Inc. Annual Report Form 10-KSB 2002. https://sec.report/Document/0000950144-02007036/

2. Williams R, Rankin N, Smith T, et al. Relationship between the humidity and temperature of inspired gas and the function of the airway mucosa. Crit Care Med 1996; 24 : 1920-1929.

3. Ritchie JE, Williams AB, Gerard C, et al. Evaluation of a humidified nasal high-flow oxygen system, using oxygraphy, capnography and measurement of upper airway pressures. Anaesth Intensive Care 2011; 39: 1103-1110.

4. Roca O, Riera J, Torres F, et al. High-flow oxygen therapy in acute respiratory failure. Respir Care 2010; 55: 408-413.

5. Sivieri EM, Gerdes JS, Abbasi S. Effect of HFNC flow rate, cannula size, and nares diameter on generated airway pressures: an in vitro study. Pediatr Pulmonol 2013; 48: 506-514

6. Braunlich J, Kohler M, Wirtz H. Nasal highflow improves ventilation in patients with COPD. Int J Chron Obstruct Pulmon Dis 2016; 11: 1077-1085.

7. Corley A, Rickard CM, Aitken LM, et al. High-flow nasal cannulae for respiratory support in adult intensive care patients. Cochrane Database Syst Rev 2017; 5: CD010172.

8. Stéphan F, Barrucand B, Petit P, et al. High-flow nasal oxygen vs noninvasive positive airway pressure in hypoxemic patients after cardiothoracic surgery: A randomized clinical trial. JAMA 2015; 313: 2331-2339.

9. Hernandez G, Vaquero C, Colinas L, et al. Effect of postextubation high-flow nasal cannula vs noninvasive ventilation on reintubation and postextubation respiratory failure in high-risk patients: a randomized clinical trial. JAMA 2016; 316: 1565-1574

10. Girod S, Zahm JM, Plotkowski C, et al. Role of the physiochemical properties of mucus in the protection of the respiratory epithelium. Eur RespirJ 1992; 5: 477-487.

11. Heaton RW, Henderson AF, Gray BJ, et al. The bronchial response to cold air challenge: evidence for different mechanisms in normal and asthmatic subjects. Thorax 1983; 38: 506-511.

12. Barker AF. Bronchiectasis. N Engl J Med 2002; 346 : 1383-1393.

13. Vestbo J, Prescott E, Lange P. Association of chronic mucus hypersecretion with FEV1 decline and chronic obstructive pulmonary disease morbidity. Copenhagen City Heart Study Group. Am J Respir Crit Care Med 1996; 153: 1530-1535.

14. Hasani A, Chapman TH, McCool D, et al. Domiciliary humidification improves lung mucociliary clearance in patients with bronchiectasis. Chron Respir Dis 2008; 5: 81-86.

15. Moller W, Celik G, Feng S, et al. Nasal high flow clears anatomical dead space in upper airway models. J Appl Physiol (1985) 2015; 118: 1525-1532.

16. Cirio S, Piran M, Vitacca $M$, et al. Effects of heated and humidified high flow gases during high-intensity constantload exercise on severe COPD patients with ventilatory limitation. Respir Med 2016; 118: 128-132.

17. Wettstein RB, Shelledy DC, Peters JI. Delivered oxygen concentrations using low-flow and high-flow nasal cannulas. Respir Care 2005; 50: 604-609.

18. Parke R, McGuinness S, Eccleston M. Nasal high-flow therapy delivers low level positive airway pressure. BrJ Anaesth 2009; 103: 886-890.

19. Mauri T, Turrini C, Eronia N, et al. Physiologic effects of highflow nasal cannula in acute hypoxemic respiratory failure. Am J Respir Crit Care Med 2017; 195: 1207-1215.

20. Pisani L, Fasano L, Corcione $\mathrm{N}$, et al. Change in pulmonary mechanics and the effect on breathing pattern of high flow oxygen therapy in stable hypercapnic COPD. Thorax 2017; 72: 373-375

21. Fraser JF, Spooner AJ, Dunster KR, et al. Nasal high flow oxygen therapy in patients with COPD reduces respiratory rate and tissue carbon dioxide while increasing tidal and end-expiratory lung volumes: a randomised crossover trial. Thorax 2016; 71 : 759-761
22. Kohnlein T, Windisch W, Kohler D, et al. Non-invasive positive pressure ventilation for the treatment of severe stable chronic obstructive pulmonary disease: a prospective, multicentre, randomised, controlled clinical trial. Lancet Respir Med 2014; 2: 698-705.

23. Murphy PB, Rehal S, Arbane G, et al. Effect of home noninvasive ventilation with oxygen therapy vs oxygen therapy alone on hospital readmission or death after an acute COPD exacerbation: a randomized clinical trial. JAMA 2017; 317: 2177-2186

24. Stripoli T, Spadaro S, Di Mussi R, et al. High-flow oxygen therapy in tracheostomized patients at high risk of weaning failure. Ann Intensive Care 2019; 9: 4.

25. Frat J-P, Thille AW, Mercat A, et al. High-flow oxygen through nasal cannula in acute hypoxemic respiratory failure. $N$ Engl J Med 2015; 372: 2185-2196.

26. Hernandez G, Vaquero C, Gonzalez P, et al. Effect of postextubation high-flow nasal cannula vs conventional oxygen therapy on reintubation in low-risk patients: a randomized clinical trial. JAMA 2016; 315: 1354-1361.

27. Spoletini G, Mega C, Pisani L, et al. High-flow nasal therapy vs standard oxygen during breaks off noninvasive ventilation for acute respiratory failure: a pilot randomized controlled trial. J Crit Care 2018; 48: 418-425.

28. Miguel-Montanes R, Hajage D, Messika J, et al. Use of highflow nasal cannula oxygen therapy to prevent desaturation during tracheal intubation of intensive care patients with mild-to-moderate hypoxemia. Crit Care Med 2015; 43: 574-583.

29. Douglas N, Ng I, Nazeem F, et al. A randomised controlled trial comparing high-flow nasal oxygen with standard management for conscious sedation during bronchoscopy. Anaesthesia 2018; 73: 169-176.

30. Azoulay E, Lemiale V, Mokart D, et al. Effect of high-flow nasal oxygen vs standard oxygen on 28-day mortality in immunocompromised patients with acute respiratory failure: the HIGH randomized clinical trial. JAMA 2018; 320: 2099-2107.

31. Frat JP, Ragot S, Girault C, et al. Effect of non-invasive oxygenation strategies in immunocompromised patients with severe acute respiratory failure: a post-hoc analysis of a randomised trial. Lancet Respir Med 2016; 4: 646-652.

32. Boles JM, Bion J, Connors A, et al. Weaning from mechanical ventilation. Eur Respir J 2007; 29: 1033-1056.

33. Davidson C, Banham S, Elliott M, et al. British Thoracic Society/Intensive Care Society Guideline for the ventilatory management of acute hypercapnic respiratory failure in adults. BMJ Open Respir Res 2016; 3: e000133.

34. Berbenetz N, Wang Y, Brown J, et al. Non-invasive positive pressure ventilation (CPAP or bilevel NPPV) for cardiogenic pulmonary oedema. Cochrane Database Syst Rev 2019; 4: CD005351.

35. Davidson AC, Banham S, Elliott M, et al. BTS/ICS guideline for the ventilatory management of acute hypercapnic respiratory failure in adults. Thorax 2016; 71: Suppl. 2, ii1-ii35.

36. Braunlich J, Beyer D, Mai D, et al. Effects of nasal high flow on ventilation in volunteers, COPD and idiopathic pulmonary fibrosis patients. Respiration 2013; 85: 319-325.

37. Braunlich J, Dellweg D, Bastian A, et al. Nasal high-flow versus noninvasive ventilation in patients with chronic hypercapnic COPD. Int J Chron Obstruct Pulmon Dis 2019; 14: 1411-1421

38. Cortegiani A, Longhini F, Carlucci A, et al. High-flow nasal therapy versus noninvasive ventilation in COPD patients with mild-to-moderate hypercapnic acute respiratory failure: study protocol for a noninferiority randomized clinical trial. Trials 2019; 20: 450

39. Ricard JD, Dib F, Esposito-Farese M, et al. Comparison of high flow nasal cannula oxygen and conventional oxygen therapy on ventilatory support duration during acute-onchronic respiratory failure: study protocol of a multicentre, randomised, controlled trial. The 'HIGH-FLOW ACRF' study. BMJ Open 2018; 8: e022983.
Suggested

\section{answers}

1. d.

2. c.

3. d.

4. b. 
40. Storgaard LH, Hockey HU, Laursen BS, et al. Long-term effects of oxygen-enriched high-flow nasal cannula treatment in COPD patients with chronic hypoxemic respiratory failure. Int J Chron Obstruct Pulmon Dis 2018 13: 1195-1205.

41. Prieur G, Medrinal C, Combret Y, et al. Nasal high flow does not improve exercise tolerance in COPD patients recovering from acute exacerbation: A randomized crossover study. Respirology 2019; 24: 1088-1094.

42. Tung LF, Shen SY, Shih HH, et al. Effect of high-flow nasal therapy during early pulmonary rehabilitation in patients with severe AECOPD: a randomized controlled study. Respir Res 2020; 21: 84

43. Wilson ME, Mittal A, Dobler CC, et al. High-flow nasal cannula oxygen in patients with acute respiratory failure and do-notintubate or do-not-resuscitate orders: a systematic review. J Hosp Med 2020; 15: 101-106.
44. Messika J, Ben Ahmed K, Gaudry S, et al. Use of high-flow nasal cannula oxygen therapy in subjects with ARDS: a 1-year observational study. Respir Care 2015; 60: 162-169.

45. Vianello A, Arcaro G, Molena B, et al. High-flow nasal cannula oxygen therapy to treat patients with hypoxemic acute respiratory failure consequent to SARS-CoV-2 infection. Thorax 2020; 75: 998-1000.

46. Hui DS, Chow BK, Lo T, et al. Exhaled air dispersion during high-flow nasal cannula therapy versus CPAP via different masks. Eur Respir J 2019; 53: 1802339.

47. Loh NW, Tan Y, Taculod J, et al. The impact of high-flow nasal cannula (HFNC) on coughing distance: implications on its use during the novel coronavirus disease outbreak. Can J Anaesth 2020; 67: 893-894.

48. Raboud J, Shigayeva A, McGeer A, et al. Risk factors for SARS transmission from patients requiring intubation: a multicentre investigation in Toronto, Canada. PLoS One 2010; 5: e10717. 